\title{
Sarcopenia and motoric cognitive risk syndrome: a moderated mediation model
}

\author{
Ting Zhang ${ }^{1,2 \dagger}$, Yunyun Zhang ${ }^{1,3+}$, Ziyan Lv ${ }^{1,3}$ and Jie Xiang ${ }^{1,2,3^{*}}$
}

\begin{abstract}
Background: Sarcopenia has been identified as a risk factor for cognitive impairment, and motoric cognitive risk syndrome (MCR) is a recently defined pre-dementia syndrome. It is not known whether they are related. We aimed to investigate the association and potential pathways between sarcopenia and MCR in the community elderly by establishing a moderated mediation model.

Methods: 846 community residents aged $\geq 60$ years were recruited from May 2021 to September 2021 and had a comprehensive geriatric evaluation. The diagnosis of sarcopenia followed the criteria issued by the Asian Working Group for Sarcopenia in 2019. MCR was defined as subjective cognitive decline and slow gait. Apathy symptoms and physical activity were assessed by the Apathy Evaluation Scale (AES) and the International Physical Activity Questionnaire (IPAQ). Logistic regression and moderated mediation analyses were conducted to explore the association between the four.

Results: 60 (7.1\%) had MCR among 846 participants. After full adjustment, sarcopenia (odds ratio [OR] = 3.81, 95\% confidence interval $[\mathrm{Cl}]=1.69-8.60, P=0.001)$, AES score $(\mathrm{OR}=1.09,95 \% \mathrm{Cl}=1.04-1.14, P<0.001)$, and IPAQ level $(\mathrm{OR}=0.43,95 \% \mathrm{Cl}=0.28-0.66, P<0.001)$ were associated with MCR. Apathy partially mediated the relationship between sarcopenia and MCR. Physical activity played a moderation role in the indirect pathway of the mediation model. The increase in physical activity can alleviate the indirect effect of sarcopenia on MCR.
\end{abstract}

Conclusion: We established a moderated mediation model to uncover the underlying association mechanism of sarcopenia and MCR preliminarily. These findings suggest that attention should be paid to the management of apathy and physical activity in the context of sarcopenia to prevent early dementia actively. Further validation is needed in future longitudinal studies.

Keywords: Sarcopenia, Motoric Cognitive Risk syndrome, Apathy, Physical activity, Moderated mediation

\section{Introduction}

Motoric cognitive risk syndrome (MCR) was proposed and verified in 2013, defined as cognitive complaints and slow gait in the elderly without dementia and mobility disability [1]. Slow gait and cognitive complaints have been proven to be the early risk markers for dementia $[2,3]$,

*Correspondence: 18052268386@163.com

${ }^{\dagger}$ Ting Zhang and Yunyun Zhang are co-first authors.

${ }^{1}$ Department of Rehabilitation, The Affiliated Hospital of Xuzhou Medical

University, Xuzhou 221002, Jiangsu, China

Full list of author information is available at the end of the article based on which MCR was established. Both MCR and mild cognitive impairment (MCI) are the intermediate state between normal cognitive aging and dementia [1]. Compared with MCI, the diagnosis of MCR substitutes the objective cognitive impairment criterion with a slow gait, which effectively complements the identification of individuals at high risk of dementia. Even after accounting for diagnostic overlap with MCI subtypes, MCR is related to future dementia, especially vascular dementia [1]. In addition, although MCR is a motor-based syndrome, the ability to predict the risk of dementia transformation once diagnosed with MCR is derived from the degree of 
cognitive impairment rather than motor disorder [4]. Prospective studies have shown that MCR is an independent risk factor for dementia $[1,4,5]$ and death [6].

Sarcopenia is an age-related loss in skeletal muscle mass accompanied by decreased muscle strength or physical performance [7]. Recent studies have shown that, except for influence on physical function, sarcopenia is related to cognitive impairment in the elderly $[8,9]$. Beeri et al. [10] reported sarcopenia as predictors of latelife Alzheimer's disease (AD), MCI, and cognitive decline in older adults. Diagnostic components of sarcopenia (muscle mass [11, 12], muscle strength [13], and physical function $[13,14])$ were also independently associated with cognitive impairment to varying degrees. Moreover, among people who already had cognitive impairment, Sugimoto et al. [15] found an increased prevalence of sarcopenia from amnestic MCI to AD. However, these researches focused on dementia and MCI. No study to date has explored the association of MCR with sarcopenia. Whether sarcopenia is involved in the pathological mechanism of MCR remains an open issue.

Traditionally, apathy is a neuropsychiatric syndrome of primary motivational loss, which cannot attribute to emotional disturbance, intellectual damage, or attenuate consciousness [16]. Lack of motivation is the core symptom, evidenced by the quantitative reduction in goaldirected behaviors [17]. Apathy was once considered a sub-symptom of depression, but subsequent studies have found the difference in the disease course, clinical symptoms, and treatment methods $[18,19]$. Apathy is highly prevalent in many neurological disorders and is associated with cognitive impairment in these patients [20-22]. Meanwhile, longitudinal studies documented that apathy also correlated to cognitive decline [23] and increased risk of dementia [24] among the community elderly. Apathy predicted incident MCR in community older adults [25]. On the other hand, handgrip strength and other sarcopenia-related physical performances were associated with affective functions of subjects from the specialized dementia outpatient clinic, such as apathy [26]. Considering its association with sarcopenia and MCR respective, it is suspected that apathy may be a mediating factor between the two.

Physical activity affects physical and cognitive function. Inactivity contributes to the development of sarcopenia [27]. Additionally, decreased daytime motor activity was found in AD patients with apathy [28], and low vitality was considered as the dementia-specific risk factor for sarcopenia [15]. In contrast, physical exercise improved sarcopenia status [29], and positively affected cognitive function in both older adults with and without cognitive impairment [30, 31]. These studies suggest that physical activity may moderate the relationship between sarcopenia and cognitive impairment.

Current treatments for dementia are often unsatisfactory, so there is a need to focus on the early stages of cognitive impairment to develop effective prevention strategies. As a pre-dementia syndrome, MCR is reversible before further cognitive decline [32]. Sarcopenia is also an invertible geriatric syndrome. Resistance training and amino acid supplementation can effectively improve sarcopenia status [29, 33, 34]. Therefore, exploring the relationship between sarcopenia, apathy, and MCR may provide a basis for effective intervention strategies before clinical adverse events. This study hypothesized that sarcopenia correlated to MCR, in which apathy played a mediating role and physical activity worked as a moderator.

\section{Methods \\ Study population}

We invited community residents, who were $\geq 60$ years and independent in basic daily life, to participate in the study from May 2021 to September 2021 in Xuzhou, China. The final analytic sample consisted of 846 participants after excluding 101 subjects. The exclusions were as follows: 17 participants failed to measure body composition analysis due to cardiac stents or pacemakers; 6 participants were unable to perform the physical function tests due to severe lumbar disc herniation or hip disease; 78 participants were lack of covariates. No participants in this study had dementia, Parkinson's disease, multiple sclerosis, and hemiplegia. All participants provided informed consent. The Ethics Committee of the Affiliated Hospital of Xuzhou Medical University approved this research, and the study methods followed the principles of the Declaration of Helsinki.

\section{Definition of MCR}

The operational definition of MCR is the presence of subjective cognitive decline (SCD) and slow gait in subjects without dementia and mobility disability. The diagnostic procedure is as follows $[1,35]$ :

1. SCD is determined based on the positive response to a memory item in the 15-item Geriatric Depression Scale (GDS) [36], whose standardized question is "Do you feel you have more problems with memory than most?".

2. Slow gait is defined as the usual walking speed one standard deviation or more below age- and gender-specific mean values established in the study population (Table 1).

3. Absence of mobility disability refers to no difficulty in dressing, eating, bathing, going to the toilet, continence, and transferring [37]. 
Table 1 The gait speed cut-off values of slow gait for Motoric Cognitive Risk syndrome

\begin{tabular}{lll}
\hline Age group (y) & Male $(\mathrm{m} / \mathbf{s})$ & Female $(\mathrm{m} / \mathbf{s})$ \\
\hline $60-64$ & 1.064 & 0.935 \\
$65-69$ & 0.872 & 0.845 \\
$70-74$ & 0.814 & 0.781 \\
$75-79$ & 0.775 & 0.729 \\
$80-84$ & 0.715 & 0.585 \\
$85+$ & 0.610 & 0.512 \\
\hline
\end{tabular}

4. There is no confirmed dementia. In addition, the Clinical Dementia Rating Scale (CDRS) score is less than or equal to 0.5 [38].

\section{Diagnosis of sarcopenia}

Based on the diagnostic criteria issued by the Asian Working Group of Sarcopenia (AWGS) in 2019, the definition of sarcopenia is low muscle mass plus low muscle strength or poor physical performance [39]. Muscle mass was measured by direct segmental multi-frequency bioelectrical impedance analysis (InBody270; Biospace Co., Ltd, Seoul, Korea). The skeletal muscle index (SMI) is the relative skeletal muscle mass of limbs divided by the square of height. Low muscle mass means SMI is lower than $7.0 \mathrm{~kg} / \mathrm{m}^{2}$ in men or $5.7 \mathrm{~kg} / \mathrm{m}^{2}$ in women.

Muscle strength was evaluated using a handheld dynamometer (EH101; Xiangshan Inc., Guangdong, China). Participants were asked to use their dominant hands to exert the best effort twice, recording the maximum value for analysis. Low muscle strength refers to handgrip strength $<28 \mathrm{~kg}$ in men or $<18 \mathrm{~kg}$ in women.

AWGS 2019 recommends defining low physical performance based on the Short-Physical Performance Battery (SPPB), or 6-m gait speed test, or five-times sit-to-stand test (FTSST). The diagnosis of MCR already contains a slow gait. To avoid the overlapping effect of gait speed on the relationship between sarcopenia and MCR, we used FTSST without walking speed test as the index of physical function in sarcopenia. Participants were asked to stand up from a standard armless chair five times as fast as possible, crossing their upper limbs upon the chest. They were instructed to stand up with their legs fully straightened and sit down firmly in each sit-to-stand process. The FTSST time $\geq 12 \mathrm{~s}$ is the cut-off value for poor physical performance.

\section{Assessment of apathy symptoms}

The Apathy Evaluation Scale (AES) self-rated version was used to quantify apathy symptoms [40]. The AES consists of 18 items scored on a 4-point Likert type scale, representing one of the most clinically used scales for apathy. It identifies apathy based on three areas: emotional responsiveness, thought content, and observation of activity. 37 is the cut-off score for apathy [41]. The higher the score, the more serious the apathy symptoms.

\section{Evaluation of physical activity}

We evaluated physical activity by the International Physical Activity Questionnaire (IPAQ) [42]. The scale assesses the amount of time spent on vigorous activity, moderateintensity activity, walking, and sitting during the previous seven days. Metabolic Equivalent of Energy (MET) values were assigned according to activity intensity. Physical activity levels were classified as low, medium, and high.

\section{Other measurements}

We collected basic information through a standard questionnaire, including age, gender, education level, night sleep duration, the number of prescription drugs, chronic medical conditions, smoking history, drinking history, fall history last year, self-perceived vision status, and self-perceived health status. Chronic diseases include hypertension, dyslipidemia, diabetes, gout, coronary heart disease, asthma, chronic respiratory diseases, arthritis, osteoporosis, peptic ulcer, thyroid disease, cancer. Smoking/drinking refers to smoking/drinking daily for more than three months. Self-perceived visual/ health status was to ask participants how to view their own visual/health. Ratings included poor, fair, good, and excellent, among which "poor" and "fair" meant poor selfperceived visual/health. We also measured the height and weight on-site to calculate body mass index (BMI) (weight divided by height squared). The Chinese version of the mini-mental state examination (MMSE) was used to evaluate cognitive function [43]. Nutritional status was assessed by the Mini Nutritional Assessment Short-Form (MNA-SF) [44]. The 15-item GDS was used to assess depressive symptoms [36].

\section{Statistical analyses}

Firstly, we compared characteristics between nonMCR and MCR participants. Continuous variables are expressed as mean $\pm S D$ or median (25-75 percentiles) as appropriate. Categorical variables are expressed as proportions. The independent t-test or Wilcoxon ranksum test was used for continuous variables, and the chisquare test was used for categorical variables. Secondly, univariate and multivariate logistic regression analyses were conducted to explore the association of MCR with sarcopenia, apathy, and physical activity. Finally, we estimated the moderated mediation model of sarcopenia and MCR using Mplus software. Mplus can build structural equation modelling (SEM) and allows dichotomous 
outcome variables in the model $[45,46]$. The Mplus codes for mediation models are available at the website [47]. Maximum-likelihood estimation in Mplus conducts the default logistic regression for a binary outcome variable and requires numerical integration. Therefore, the direct and indirect effects of SEM are defined based on a continuous latent response variable underlying the binary outcome [45]. Covariates included gender, age, education level, BMI, and health-related variables with statistical differences between groups. All continuous variables were centered using the group mean before the model fit to facilitate the interpretation of the results. We set 5000 repeated bootstrap samples to calculate the biascorrected 95\% confidence interval. The coefficient of the path analysis is statistically significant if the confidence interval does not contain zero, while the effect in an odds ratio metric is statistically significant if the confidence interval does not cover one. All statistical analyses were performed using IBM SPSS Statistics for Windows, version 25.0 (SPSS Inc, Chicago, Illinois, the United States) and Mplus version 8.3 (Muthén \& Muthén, Los Angeles, California, the United States). Statistical significance was a two-sided $P<0.05$.

\section{Results}

\section{Characteristics for MCR}

Among the 846 participants, 60 (7.1\%) had MCR, including $40(7.9 \%)$ females and $20(5.9 \%)$ males. Table 2 presents the characteristics of the study population grouped by MCR. Individuals with MCR tended to be older and less educated $(P<0.05)$, whereas there was no difference in gender and BMI between groups. In health-related variables, night sleep duration, number of prescription drugs, number of chronic diseases, chronic respiratory diseases, arthritis, MNA-SF, IPAQ, fall history last year, poor self-perceived vision, poor self-perceived health, GDS score, AES score, MMSE score, sarcopenia, SMI, handgrip strength, FTSST time and gait speed were significantly different between groups $(P<0.05)$.

\section{Associations of sarcopenia, apathy, and physical activity with MCR}

Sarcopenia, apathy, and physical activity were associated with MCR both in univariate and multivariate logistic regression models (Table 3). After adjustment for all covariates, sarcopenia (odds ratio $[\mathrm{OR}]=3.81,95 \%$ confidence interval $[\mathrm{CI}]=1.69-8.60, P=0.001)$ and AES score $(\mathrm{OR}=1.09,95 \% \mathrm{CI}=1.04-1.14, P<0.001)$ were positively related to MCR. Physical activity $(\mathrm{OR}=0.43,95 \%$ $\mathrm{CI}=0.28-0.66, P<0.001)$ was negatively associated with MCR.
Table 2 Characteristics of study participants according to Motoric Cognitive Risk syndrome status

\begin{tabular}{|c|c|c|c|}
\hline Characteristic & $\begin{array}{l}\text { Non-MCR } \\
(n=786)\end{array}$ & $\begin{array}{l}\text { MCR } \\
(n=60)\end{array}$ & $P$ value \\
\hline Age (years) & $71.0 \pm 8.1$ & $74.4 \pm 8.6$ & 0.002 \\
\hline \multicolumn{4}{|l|}{ Gender } \\
\hline Male (\%) & $318(40.5)$ & $20(33.3)$ & \\
\hline Female (\%) & $468(59.5)$ & $40(66.7)$ & 0.277 \\
\hline $\mathrm{BMI}\left(\mathrm{kg} / \mathrm{m}^{2}\right)$ & $24.39 \pm 3.19$ & $24.11 \pm 3.72$ & 0.516 \\
\hline \multicolumn{4}{|l|}{ Education level (\%) } \\
\hline Illiteracy & $111(14.1)$ & $19(31.7)$ & \\
\hline Primary school & $147(18.7)$ & $14(23.3)$ & \\
\hline Secondary school & $445(56.6)$ & $26(43.3)$ & \\
\hline College & $83(10.6)$ & $1(1.7)$ & 0.001 \\
\hline Night sleep duration (h) & $6.4 \pm 0.9$ & $6.0 \pm 1.4$ & 0.033 \\
\hline Smoking (\%) & $118(15.0)$ & $8(13.3)$ & 0.725 \\
\hline Drinking (\%) & $107(13.6)$ & $4(6.7)$ & 0.124 \\
\hline $\begin{array}{l}\text { Number of prescription } \\
\text { drugs }\end{array}$ & $1.0(0.0,2.0)$ & $1.5(0.0,4.0)$ & 0.001 \\
\hline $\begin{array}{l}\text { Number of chronic } \\
\text { diseases }\end{array}$ & $1.0(0.0,2.0)$ & $1.5(0.5,2.5)$ & 0.033 \\
\hline \multicolumn{4}{|l|}{ Chronic conditions (\%) } \\
\hline $\begin{array}{l}\text { chronic respiratory } \\
\text { diseases }\end{array}$ & $29(3.7)$ & $9(15.0)$ & $<0.001$ \\
\hline Hypertension & $300(38.2)$ & $23(38.3)$ & 0.980 \\
\hline diabetes & $113(14.4)$ & $12(20.0)$ & 0.237 \\
\hline Arthritis & $158(20.1)$ & $19(31.7)$ & 0.034 \\
\hline Osteoporosis & $65(8.3)$ & $2(3.3)$ & 0.264 \\
\hline MNA-SF score & $14(13,14)$ & $13(12,14)$ & 0.001 \\
\hline IPAQ (Met/week) & $2398(1716,2982)$ & $1704(940,2166)$ & $<0.001$ \\
\hline \multicolumn{4}{|l|}{ IPAQ level } \\
\hline Low & $15(1.9)$ & $11(18.3)$ & \\
\hline Medium & $575(73.2)$ & $44(73.3)$ & \\
\hline High & $196(24.9)$ & $5(8.3)$ & $<0.001$ \\
\hline Fall history last year & $55(7.0)$ & $14(23.3)$ & $<0.001$ \\
\hline $\begin{array}{l}\text { Self-perceived vision } \\
\text { status }\end{array}$ & $488(62.1)$ & $50(83.3)$ & 0.001 \\
\hline $\begin{array}{l}\text { Self-perceived health } \\
\text { status }\end{array}$ & $248(31.6)$ & $33(55.0)$ & $<0.001$ \\
\hline GDS score & $1(0,2)$ & $3(2,6)$ & $<0.001$ \\
\hline AES score & $29.5 \pm 6.9$ & $38.8 \pm 9.2$ & $<0.001$ \\
\hline MMSE score & $27(25,29)$ & $24(21,27)$ & $<0.001$ \\
\hline Sarcopenia (\%) & $76(9.7)$ & $21(35.0)$ & $<0.001$ \\
\hline $\mathrm{SMI}\left(\mathrm{kg} / \mathrm{m}^{2}\right)$ & $6.79 \pm 0.99$ & $6.37 \pm 0.86$ & $<0.001$ \\
\hline Handgrip strength (kg) & $27.23 \pm 8.94$ & $20.22 \pm 6.66$ & $<0.001$ \\
\hline FTSST time (s) & $10.60 \pm 3.04$ & $15.21 \pm 3.92$ & $<0.001$ \\
\hline Gait speed (m/s) & $1.05 \pm 0.24$ & $0.65 \pm 0.15$ & $<0.001$ \\
\hline
\end{tabular}

$B M I$ body mass index, MNA-SF Mini Nutritional Assessment Short-Form, IPAQ International Physical Activity Questionnaire, Met/week metabolic equivalent task minutes per week, GDS Geriatric Depression Scale, AES Apathy Evaluation Scale, MMSE mini-mental state examination, SMI skeletal muscle index, FTSST five-times sit-to-stand test 
Table 3 Associations of sarcopenia, apathy, and physical activity with MCR

\begin{tabular}{|c|c|c|c|c|c|c|}
\hline \multirow[t]{2}{*}{ Variables } & \multicolumn{2}{|c|}{ Unadjusted model } & \multicolumn{2}{|c|}{ Adjusted model 1} & \multicolumn{2}{|c|}{ Adjusted model 2} \\
\hline & OR $(95 \% \mathrm{Cl})$ & $P$ value & OR $(95 \% \mathrm{Cl})$ & $P$ value & OR $(95 \% \mathrm{Cl})$ & $P$ value \\
\hline Sarcopenia & $5.03(2.81,8.99)$ & $<0.001$ & $3.10(1.46,6.56)$ & 0.003 & $3.81(1.69,8.60)$ & 0.001 \\
\hline AES score & $1.15(1.11,1.19)$ & $<0.001$ & $1.09(1.04,1.14)$ & $<0.001$ & $1.09(1.04,1.14)$ & $<0.001$ \\
\hline IPAQ & $0.32(0.22,0.47)$ & $<0.001$ & $0.42(0.28,0.64)$ & $<0.001$ & $0.43(0.28,0.66)$ & $<0.001$ \\
\hline
\end{tabular}

SMI skeletal muscle index, FTSST five-times sit-to-stand test, IPAQ International Physical Activity Questionnaire, AES Apathy Evaluation Scale, CI confidence interval. Adjusted model 1 had adjusted potential confounders including age; education level; night sleep duration; Mini Nutritional Assessment Short-Form score; the number of prescription drugs; the number of chronic diseases; chronic respiratory diseases; Arthritis; Geriatric Depression Scale score; self-perceived vision status; self-perceived health status; fall history last year. Adjusted model 2 had adjusted all the covariates (model 2 plus gender; body mass index; smoking; drinking; hypertension; diabetes; osteoporosis)

\section{Mediation model}

Path analysis showed that the pathway of sarcopenia to apathy, apathy to MCR, and sarcopenia to MCR were statistically significant (all $P<0.01$ ). The point estimate of the indirect effect was 0.314 (bootstrap estimated $95 \% \mathrm{CI}=0.116-0.559, P=0.007)$. The indirect effect odds ratio was 1.369 (bootstrap estimated 95\% $\mathrm{CI}=1.123-1.750, \quad P<0.001)$, which meant that the odds ratio of MCR prevalence increased by 0.369 due to the mediational mechanism of apathy. The direct effect and the direct effect odds ratio of sarcopenia on MCR were 1.456 (bootstrap estimated 95\% CI $=0.442-$ 2.282, $P=0.002$ ) and 4.289 (bootstrap estimated $95 \%$ $\mathrm{CI}=1.556-9.799, \quad P=0.121$ ), respectively (Table 4 , Fig. 1a). This indicated that apathy partially mediated the association between sarcopenia and MCR. The ratio of the indirect effect odds ratio to the direct effect odds ratio is $31.92 \%$.

\section{Moderated mediation model}

We first tested the moderation effects of physical activity on all of the sarcopenia-apathy path, the apathy-MCR path, and the direct sarcopenia-MCR path. The result revealed that physical activity only significantly moderated the sarcopenia-apathy path. Therefore, we refit the model to examine the moderation effect of physical activity on the first half of the mediation path. As shown in the Table 4 and Fig. $1 \mathrm{~b}$, the point estimate of moderating effect was -1.958 (bootstrap estimated $95 \% \mathrm{CI}=-3.438$ $0.272, P=0.013)$. This suggested that physical activity moderated the indirect pathways of sarcopenia to MCR.

We further analyzed the conditional indirect effects of sarcopenia on MCR under different levels of physical activity. From low to medium physical activity level, the indirect effects (low: $\beta=0.393$, bootstrap estimated 95\% $\mathrm{CI}=0.161-0.696$; medium: $\beta=0.203$, bootstrap estimated $95 \% \mathrm{CI}=0.026-0.428)$ and indirect effects

Table 4 Path analysis of the mediation and moderated mediation model using structural equation modelling

\begin{tabular}{|c|c|c|c|c|c|c|c|c|}
\hline $\begin{array}{l}\text { Dependent } \\
\text { variable }\end{array}$ & Independent variable & Effect & Estimate & SE & Est/SE & $P$ value & BootLLCI & BootLLCI \\
\hline \multicolumn{9}{|c|}{ Mediation model } \\
\hline Apathy & Sarcopenia & & 3.241 & 0.896 & 3.615 & $<0.001$ & 1.467 & 4.954 \\
\hline \multirow[t]{6}{*}{ MCR } & Sarcopenia & & 1.456 & 0.476 & 3.061 & 0.002 & 0.442 & 2.282 \\
\hline & Apathy & & 0.097 & 0.025 & 3.813 & $<0.001$ & 0.043 & 0.140 \\
\hline & & Direct effect & 1.456 & 0.476 & 3.061 & 0.002 & 0.442 & 2.282 \\
\hline & & Direct effect odds ratio & 4.289 & 2.770 & 1.549 & 0.121 & 1.556 & 9.799 \\
\hline & & Indirect effect & 0.314 & 0.117 & 2.685 & 0.007 & 0.116 & 0.559 \\
\hline & & Indirect effect odds ratio & 1.369 & 0.170 & 8.031 & $<0.001$ & 1.123 & 1.750 \\
\hline \multicolumn{9}{|c|}{ Moderated mediation model } \\
\hline \multirow[t]{3}{*}{ Apathy } & Sarcopenia & & 2.097 & 0.926 & 2.265 & 0.024 & 0.329 & 3.908 \\
\hline & Physical activity & & -1.234 & 0.276 & -4.467 & $<0.001$ & -1.766 & -0.673 \\
\hline & Sarcopenia*Physical activity & & -1.958 & 0.791 & -2.475 & 0.013 & -3.438 & -0.272 \\
\hline \multirow[t]{2}{*}{ MCR } & Sarcopenia & & 1.456 & 0.476 & 3.061 & 0.002 & 0.442 & 2.282 \\
\hline & Apathy & & 0.097 & 0.025 & 3.813 & $<0.001$ & 0.043 & 0.140 \\
\hline
\end{tabular}

$M C R$ motoric cognitive risk syndrome, SE standard error, Est/SE estimate divided by the standard error. BootLLCI / BootLLCI, lower/upper 2.5\% of bias-corrected confidence interval derived from bootstrap estimates. Adjusted for gender, body mass index, and all potential confounding factors 
a.

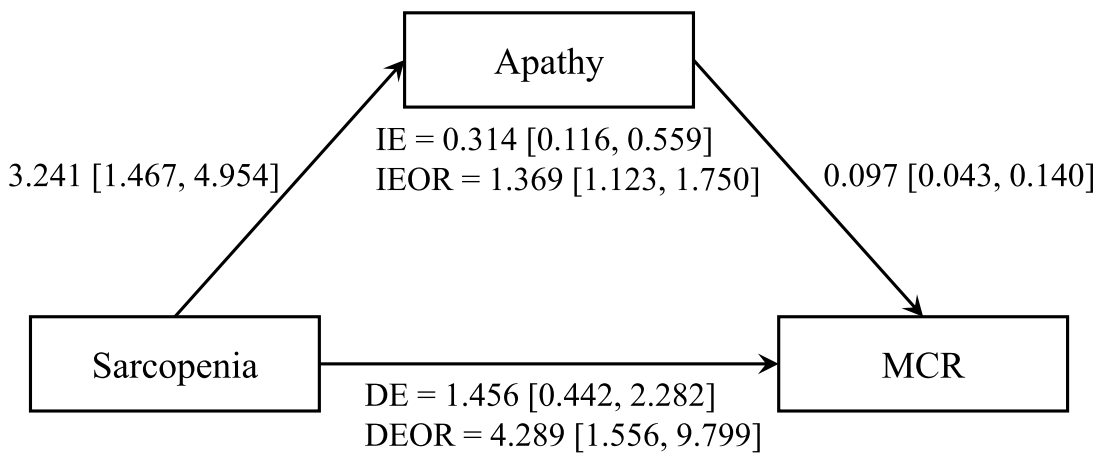

b.

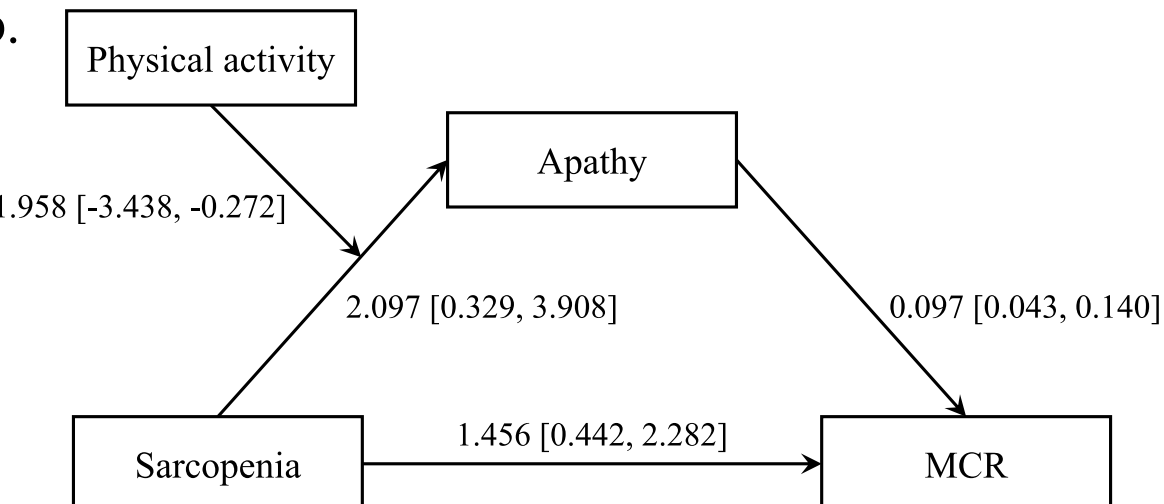

Fig. 1 Mediation and moderated mediation path diagram. Panel a, the mediating effect of apathy on the relationship between sarcopenia and MCR. Panel $b$, the moderating effect of physical activity on the mediation model. The effect values and their corresponding confidence intervals between variables are marked next to the arrow. DE, direct effect; IE, Indirect effect; DEOR/IEOR, direct/indirect effect odds ratio

odds ratio (low: $\mathrm{OR}=1.482$, bootstrap estimated $95 \%$ $\mathrm{CI}=1.175-2.006$; medium: $\mathrm{OR}=1.225$, bootstrap estimated $95 \% \mathrm{CI}=1.027-1.534$ ) were significant, and the effect size decreased with increasing physical activity level. When the physical activity was high, the indirect effects $(\beta=0.013$, bootstrap estimated $95 \% \mathrm{CI}=-0.285-$ $0.309)$ and indirect effects odds ratio $(\mathrm{OR}=1.014$, bootstrap estimated $95 \% \mathrm{CI}=0.752-1.362)$ of sarcopenia on MCR were both insignificant. Whether in an odds ratio metric or not, the differences in indirect effects between the three groups were all statistically significant (Table 5). It could be conceived that physical activity alleviated the indirect effect of sarcopenia on MCR, and the indirect effect became in-exist under the high physical activity level.

\section{Discussion}

The study examined the association between sarcopenia and MCR, and further revealed the underlying mechanisms of apathy and physical activity in the association by establishing a moderated mediation model. We found a positive connection between sarcopenia and MCR.
Apathy partially mediated the effect of sarcopenia on MCR, and physical activity moderated the indirect pathway in the mediation model. The results supported the possibility of modifying the effects of sarcopenia on MCR by altering apathy and physical activity.

A systematic review of more than 20,000 older adults in 17 countries reported the prevalence of MCR ranging from $5.3 \%$ to $15.5 \%$, with a pooled prevalence of $9.7 \%$ [48]. The prevalence of MCR in this study was $7.1 \%$, somewhat lower than that previously reported. One possible reason is that our diagnosis of SCD was relatively strict. Older people were considered to have SCD only when they felt they had more memory problems than most people. By contrast, participants were diagnosed with SCD when their self-perceived memory was either poor or worse than before in other researches [6, 49]. An alternative explanation is that our study participants were relatively healthy community residents rather than outpatients or inpatients, which may underestimate the prevalence of MCR.

As far as we know, our study is the first to identify the relationship between sarcopenia and MCR. The results 
Table 5 The conditional indirect effects under different levels of physical activity

\begin{tabular}{|c|c|c|c|c|c|c|}
\hline Indirect effect scale & $\begin{array}{l}\text { Physical activity } \\
\text { level }\end{array}$ & $\begin{array}{l}\text { Comparison between } \\
\text { levels }\end{array}$ & Estimate & SE & BootLLCI & BootLLCI \\
\hline \multirow[t]{6}{*}{$\beta$} & Low & & $0.393^{\mathrm{a}}$ & 0.141 & 0.161 & 0.696 \\
\hline & Medium & & $0.203^{\mathrm{a}}$ & 0.104 & 0.026 & 0.428 \\
\hline & High & & 0.013 & 0.148 & -0.285 & 0.309 \\
\hline & & Medium vs Low & $-0.190^{a}$ & 0.100 & -0.400 & -0.018 \\
\hline & & High vs Medium & $-0.190^{a}$ & 0.100 & -0.400 & -0.018 \\
\hline & & High vs Low & $-0.380^{\mathrm{a}}$ & 0.199 & -0.801 & -0.036 \\
\hline \multirow[t]{6}{*}{ OR } & Low & & $1.482^{\mathrm{a}}$ & 0.227 & 1.175 & 2.006 \\
\hline & Medium & & $1.225^{\mathrm{a}}$ & 0.133 & 1.027 & 1.534 \\
\hline & High & & 1.014 & 0.152 & 0.752 & 1.362 \\
\hline & & Medium vs Low & $-0.256^{\mathrm{a}}$ & 0.163 & -0.628 & -0.022 \\
\hline & & High vs Medium & $-0.212^{\mathrm{a}}$ & 0.104 & -0.425 & -0.022 \\
\hline & & High vs Low & $-0.468^{\mathrm{a}}$ & 0.266 & -1.054 & -0.044 \\
\hline
\end{tabular}

SE standard error. BootLLCI / BootLLCl, lower/upper 2.5\% of bias-corrected confidence interval derived from bootstrap estimates. The low, medium, and high values of

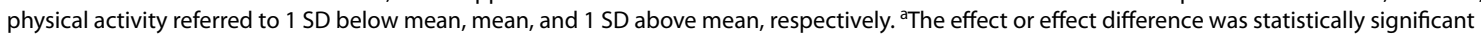

showed that sarcopenia was independently associated with a nearly threefold increased risk of MCR in the community elderly. There has been no comparable research to date on the relationship between sarcopenia and MCR. Nevertheless, the associations of sarcopenia with cognitive impairment and dementia have been controversially reported. A recent meta-analysis reported that sarcopenia was related to cognitive impairment independent of their different operational definitions and study population [9]. However, the exact mechanisms involved have not been determined. The association between sarcopenia and MCR in this study seemed to be partly explained from the perspective of physical function. Although we chose FTSST instead of the usual gait speed test in the diagnosis of sarcopenia, gait speed was significantly lower in participants with sarcopenia than normal individuals $(0.76 \mathrm{~m} / \mathrm{s}$ vs. $1.06 \mathrm{~m} / \mathrm{s}, P<0.001)$. Meanwhile, slow gait is also a motor manifestation of MCR. Thus, the link between sarcopenia and MCR may be driven by slow gait to some extent. Besides, the association between sarcopenia and motoric cognitive impairment further supported sarcopenia as a contributing factor to cognitive decline.

The mediation analysis showed that apathy was positively related to sarcopenia and MCR, and partially mediated the effect of sarcopenia on MCR. Ceide et al. [25] reported that apathy predicted future MCR but not MCI in community older adults. They also found GDS score became MCR-independent after removing the 3-item subscale of apathy in GDS [25]. A prospective study showed that apathy rather than depression was most strongly related to subsequent weight loss in
AD patients [50]. Several common underlying risk factors may explain the serial relationships. Firstly, malnutrition is a recognized risk factor for sarcopenia [51] and cognitive impairment [52] in older adults. Among older adults with mild cognitive decline, nutritional status was also independently associated with apathy [53]. In this study, The MNA-SF score of participants with MCR or sarcopenia or apathy was significantly lower than that of normal individuals (all $P<0.001$ ). Secondly, there may be hormonal dysregulation. Insulin is involved in skeletal muscle metabolism as a synthetic metabolic hormone [54]. Besides, abnormal glucose metabolism caused by insulin resistance is considered a vital pathological mechanism of $\mathrm{AD}[55]$. Burns et al. $[56,57]$ found that increased peripheral insulin was related to reduced lean mass, cognitive dysfunction, and brain atrophy in early AD patients. Thirdly, age-related inflammatory abnormalities can be a shared underlying factor. A longitudinal cohort study found that interleukin 6 and C-reactive protein (CRP) contributed to appendicular skeletal muscle loss in healthy individuals[58]. Meanwhile, Sathyan et al. [59] prospectively clarified that the overexpression of interleukin 10 was associated with incident MCR. A population-based cohort study found increased CRP levels remained associated with apathy symptoms after adjustment for demographics and depressive symptoms [60].

A moderated mediation model showed that physical activity moderated the association between sarcopenia and apathy. The indirect effect of sarcopenia on MCR weakened as physical activity levels increased. Physical inactivity is a motor characteristic of sarcopenia and apathy. It can also lead to cerebral hypoperfusion 
resulting in cognitive impairment [61]. In addition, myokines molecules secreted by skeletal muscles during exercise can regulate cerebral functions, including emotion and cognition [62]. Therefore, physical exercise supported the existence of muscle-brain crosstalk [63]. Our study suggested that physical exercise could buffer or even counteract the adverse effects of sarcopenia on apathy. Therefore, increasing physical activity is not only an essential means to improve sarcopenia status and apathy symptoms but also an effective strategy to prevent sarcopenia from promoting early dementia. Future research could investigate whether physical activity improves cognitive function in patients with sarcopenia.

There are some limitations to this study. First, the research only preliminarily discussed the mechanism of sarcopenia on MCR based on a cross-sectional design. The reverse connection between MCR and sarcopenia can also exist. Future longitudinal studies will provide strong evidence of causality. Second, the total effect is not exactly the sum of the indirect effect and the direct effect in the traditional mediation analysis with a binary outcome variable, so we did not directly compare the indirect effect with the total effect. Additionally, it could not be ruled out other potential mediators that have not yet been explored. Third, some key variables in this study relied heavily on the participants' self-report, such as apathy, physical activity, and subjective cognitive decline. We admitted that it was difficult to evaluate these latent variables with objective methods. Participants may have recall bias and exaggeration or concealment in their statements, leading to deviations between the measurements and the actual situation. Finally, the distribution of our sample population was relatively limited, which required multi-center studies for further verification. However, we believed that the sample size of this study was enough by calculating the statistical power. The univariate logistic regression of MCR on sarcopenia with a sample size of 846 observations (of which $89 \%$ were non-sarcopenia and $11 \%$ were sarcopenia) achieved $95 \%$ power at a significance level of 0.05 and an odds ratio of 5.023 .

\section{Conclusions}

Our work initially investigated the association and potential mechanism between sarcopenia and MCR. Sarcopenia was positively correlated with MCR. Sarcopenia can promote MCR through apathy symptoms, while physical activity moderated this association to some extent. Exploring these mediation pathways can provide theoretical guidance for early detection and intervention of at-risk individuals in the community elderly. At the same time, the effect mechanism of apathy and physical activity emphasizes the prevention of early dementia in the context of combining physical and mental management, such as the promotion of physical activity, nutritional support, psycho-behavioral therapy (e.g., development of interests, horticultural activity). Further longitudinal studies and intervention studies are needed to clarify the mechanisms involved.

\begin{abstract}
Abbreviations
MCR: Motoric cognitive risk; AES: Apathy evaluation scale; IPAQ: International physical activity questionnaire; OR: Odds ratio; $\mathrm{Cl}$ : Confidence interval; $\mathrm{MCl}$ : Mild cognitive impairment; AD: Alzheimer's disease; SCD: Subjective cognitive decline; CDRS: Clinical dementia rating scale; AWGS: Asian Working Group of Sarcopenia; SMI: Skeletal muscle index; FTSST: Five-times sit-to-stand test; MET: Metabolic equivalent of energy; BMI: Body mass index; MMSE: Mini-mental state examination; MNA-SF: Mini nutritional assessment short-form; GDS: Geriatric depression scale; SEM: Structural equation modelling; CRP: C-reactive protein.
\end{abstract}

\section{Acknowledgements}

All authors gratefully acknowledge all study participants for voluntary participation and the community committee staff for friendly support.

\section{Authors' contributions}

TZ and JX participated in the study concept and design, the analysis and interpretation of the data, and the drafting of the manuscript. YY and ZY contributed to the acquisition of data and provided statistical guidance. All the authors revised and approved the final manuscript.

\section{Funding}

This work was supported by the National Key Research and Development Program of the Ministry of Science and Technology of the People's Republic of China (No. 2020YFC2006604).

\section{Availability of data and materials}

The datasets used and/or analysed during the current study are available from the corresponding author on reasonable request.

\section{Declarations}

Ethics approval and consent to participate

All participants provided informed consent. The Ethics Committee of the Affiliated Hospital of Xuzhou Medical University approved this research, and the study methods followed the principles of the Declaration of Helsinki.

Consent for publication

Not applicable

Competing interests

The authors declare that they have no competing interests.

\section{Author details}

${ }^{1}$ Department of Rehabilitation, The Affiliated Hospital of Xuzhou Medical University, Xuzhou 221002, Jiangsu, China. ${ }^{2}$ Medical Technology School, Xuzhou Medical University, Xuzhou 221004, Jiangsu, China. ${ }^{3}$ The Second School of Clinical Medicine, Xuzhou Medical University, Xuzhou 221004, Jiangsu, China.

Received: 24 October 2021 Accepted: 27 January 2022

Published online: 19 February 2022

\section{References}

1. Verghese J, Wang CL, Lipton RB, et al. Motoric Cognitive Risk Syndrome and the Risk of Dementia. Journals of Gerontology Series a-Biological Sciences and Medical Sciences. 2013:68(4):412-8. 
2. Verghese J, Lipton $\mathrm{RB}$, Hall $\mathrm{CB}$, et al. Abnormality of gait as a predictor of non-Alzheimer's dementia. N Engl J Med. 2002;347(22):1761-8.

3. Amariglio RE, Becker JA, Carmasin J, et al. Subjective cognitive complaints and amyloid burden in cognitively normal older individuals. Neuropsychologia. 2012;50(12):2880-6.

4. Verghese J, Wang CL, Bennett DA, et al. Motoric cognitive risk syndrome and predictors of transition to dementia: A multicenter study. Alzheimers \& Dementia. 2019;15(7):870-7.

5. Beauchet $\mathrm{O}$, Sekhon $\mathrm{H}$, Launay $\mathrm{CP}$, et al. Pre-Dementia Stages and Incident Dementia in the NuAge Study. J Alzheimers Dis. 2021;80(4):1465-70.

6. Ayers $E$, Verghese J. Motoric cognitive risk syndrome and risk of mortality in older adults. Alzheimers Dement. 2016;12(5):556-64.

7. Cruz-Jentoft AJ, Bahat G, Bauer J, et al. Sarcopenia: revised European consensus on definition and diagnosis. Age Ageing. 2019;48(1):16-31.

8. Szlejf C, Suemoto CK, Lotufo PA, et al. Association of Sarcopenia With Performance on Multiple Cognitive Domains: Results From the ELSA-Brasil Study. J Gerontol A Biol Sci Med Sci. 2019;74(11):1805-11.

9. Peng TC, Chen WL, Wu LW, et al. Sarcopenia and cognitive impairment: A systematic review and meta-analysis. Clin Nutr. 2020;39(9):2695-701.

10. Beeri MS, Leugrans SE, Delbono O, et al. Sarcopenia is associated with incident Alzheimer's dementia, mild cognitive impairment, and cognitive decline. J Am Geriatr Soc. 2021;69(7):1826-35.

11. Kohara K, Okada Y, Ochi M, et al. Muscle mass decline, arterial stiffness, white matter hyperintensity, and cognitive impairment: Japan Shimanami Health Promoting Program study. J Cachexia Sarcopenia Muscle. 2017:8(4):557-66.

12. Bae S, Shimada H, Park H, et al. Association between body composition parameters and risk of mild cognitive impairment in older Japanese adults. Geriatr Gerontol Int. 2017;17(11):2053-9.

13. Chou MY, Nishita Y, Nakagawa T, et al. Role of gait speed and grip strength in predicting 10-year cognitive decline among communitydwelling older people. Bmc Geriatrics. 2019;19:1-11.

14. Moon JH, Moon JH, Kim KM, et al. Sarcopenia as a predictor of future cognitive impairment in older adults. Journal of Nutrition Health \& Aging. 2016:20(5):496-502.

15. Sugimoto T, Ono R, Murata $S$, et al. Prevalence and associated factors of sarcopenia in elderly subjects with amnestic mild cognitive impairment or Alzheimer disease. Curr Alzheimer Res. 2016;13(6):718-26.

16. Marin RS. Apathy: a neuropsychiatric syndrome. J Neuropsychiatry Clin Neurosci. 1991;3(3):243-54.

17. Levy R, Dubois B. Apathy and the functional anatomy of the prefrontal cortex-basal ganglia circuits. Cereb Cortex. 2006;16(7):916-28.

18. Ishii S, Weintraub N, Mervis JR. Apathy: a common psychiatric syndrome in the elderly. J Am Med Dir Assoc. 2009;10(6):381-93.

19. Holthoff VA, Beuthien-Baumann B, Kalbe E, et al. Regional cerebral metabolism in early Alzheimer's disease with clinically significant apathy or depre ssion. Biol Psychiatry. 2005;57(4):412-21.

20. Vilalta-Franch J, Calv ${ }^{\cdots \otimes}$-Perxas L, Garre-Olmo J, et al. Apathy syndrome in Alzheimer's disease epidemiology: prevalence, incidence, persistence, and risk and mortality factors. J Alzheimers Dis. 2013;33(2):535-43.

21. den Brok MG, van Dalen JW, van Gool WA, et al. Apathy in Parkinson's disease: A systematic review and meta-analysis. Mov Disord. 2015;30(6):759-69.

22. Tay J, Morris RG, Markus HS. Apathy after stroke: Diagnosis, mechanisms, consequences, and treatment. Int J Stroke. 2021;16(5):510-8.

23. Clarke DE, Ko JY, Lyketsos C, et al. Apathy and cognitive and functional decline in community-dwelling older adults: results from the Balt imore ECA longitudinal study. Int Psychogeriatr. 2010;22(5):819-29.

24. van Dalen JW, Van Wanrooij LL, Moll van Charante EP, et al. Apathy is associated with incident dementia in community-dwelling older people. Neurology. 2018;90(1):e82-9.

25. Ceide ME, Warhit A, Ayers El, et al. Apathy and the Risk of Predementia Syndromes in Community-Dwelling Older Adults. J Gerontol B Psychol Sci Soc Sci. 2020;75(7):1443-50.

26. Ohta Y, Nomura E, Hatanaka N, et al. Female dominant association of sarcopenia and physical frailty in mild cognitive impairment and Alzheimer's disease. J Clin Neurosci. 2019;70:96-101.

27. Paddon-Jones D, Sheffield-Moore M, Cree MG, et al. Atrophy and impaired muscle protein synthesis during prolonged inactivity and stress. J Clin Endocrinol Metab. 2006;91(12):4836-41.
28. David R, Mulin E, Friedman L, et al. Decreased daytime motor activity associated with apathy in Alzheimer disease: an actigraphic study. Am J Geriatr Psychiatry. 2012;20(9):806-14.

29. Vikberg S, Sörlén N, Brandén L, et al. Effects of Resistance Training on Functional Strength and Muscle Mass in 70-Year-Old Individuals With Pre-sarcopenia: A Randomized Controlled Trial. J Am Med Dir Assoc. 2019;20(1):28-34.

30. Jia RX, Liang JH, Xu Y, et al. Effects of physical activity and exercise on the cognitive function of patients with Alzheimer disease: a meta-analysis. Bmc Geriatrics. 2019;19:1-14.

31. Angevaren M, Aufdemkampe G, Verhaar HJJ et al. Physical activity and enhanced fitness to improve cognitive function in older people without known cognitive impairment. Cochrane Database of Systematic Reviews. 2008;(3)

32. Merchant RA, Chan YH, Hui RJY, et al. Motoric cognitive risk syndrome, physio-cognitive decline syndrome, cognitive frailty and reversibility with dual-task exercise. Exp Gerontol. 2021;150:111362.

33. Lichtenberg T, von Stengel S, Sieber C, et al. The Favorable Effects of a High-Intensity Resistance Training on Sarcopenia in Older CommunityDwelling Men with Osteosarcopenia: The Randomized Controlled FrOST Study. Clin Interv Aging. 2019;14:2173-86.

34. Park Y, Choi JE, Hwang HS. Protein supplementation improves muscle mass and physical performance in undernourished prefrail and frail elderly subjects: a randomized, double-blind, placebo-controlled trial. Am J Clin Nutr. 2018;108(5):1026-33.

35. Semba RD, Tian $\mathrm{Q}$, Carlson MC, et al. Motoric cognitive risk syndrome: Integration of two early harbingers of dementia in older adults. Ageing research reviews. 2020;58:101022.

36. D'Ath P, Katona P, Mullan E, et al. Screening, detection and management of depression in elderly primary care attenders. I: The acceptabi lity and performance of the 15 item Geriatric Depression Scale (GDS15) and the development of short versions. Fam Pract. 1994;1 1(3):260-6.

37. Katz S, Ford AB, Moskowitz RW, et al. STUDIES OF ILLNESS IN THE AGED. THE INDEX OF ADL: A STANDARDIZED MEASURE OF BIOLOGICAL AND PSYCHOSOCI AL FUNCTION. Jama. 1963;185:914-9.

38. Hughes CP, Berg L, Danziger WL, et al. A new clinical scale for the staging of dementia. Br J Psychiatry. 1982;140:566-72.

39. Chen LK, Woo J, Assantachai P, et al. Asian Working Group for Sarcopenia: 2019 Consensus Update on Sarcopenia Diagnosis and Treatment. J Am Med Dir Assoc. 2020;21(3):300-307.e302.

40. Marin RS, Biedrzycki RC, Firinciogullari S. Reliability and validity of the Apathy Evaluation Scale. Psychiatry Res. 1991;38(2):143-62.

41. Santangelo G, Barone P, Cuoco S, et al. Apathy in untreated, de novo patients with Parkinson's disease: validation study of Apathy Evaluation Scale. J Neurol. 2014;261(12):2319-28.

42. Craig CL, Marshall AL, Sjöström M, et al. International physical activity questionnaire: 12-country reliability and validity. Med Sci Sports Exerc. 2003;35(8):1381-95.

43. Katzman R, Zhang MY, Ouang Ya Q, et al. A Chinese version of the MiniMental State Examination; impact of illiteracy in a Shanghai dementia survey. J Clin Epidemiol. 1988;41(10):971-8.

44. Kaiser MJ, Bauer JM, Ramsch C, et al. Validation of the Mini Nutritional Assessment short-form (MNA-SF): a practical tool for identification of nutritional status. J Nutr Health Aging. 2009;13(9):782-8.

45. Muthén LK, Muthén B. Mplus user's guide: Statistical analysis with latent variables, user's guide: Muthén \& Muthén; 2017.

46. Muthén BO, Muthén LK, Asparouhov T. Regression and mediation analysis using Mplus: Muthén \& Muthén Los Angeles, CA; 2017.

47. Stride CB, Gardner S, Catley N et al. (2015) 'Mplus code for mediation, moderation, and moderated mediation models', http://www.offbeat. group.shef.ac.uk/FIO/mplusmedmod.htm.

48. Verghese J, Annweiler C, Ayers E, et al. Motoric cognitive risk syndrome: multicountry prevalence and dementia risk. Neurology. 2014;83(8):718-26.

49. George CJ, Verghese J. Motoric Cognitive Risk Syndrome in Polypharmacy. J Am Geriatr Soc. 2020;68(5):1072-7.

50. Volicer L, Frijters DH, van der Steen JT. Apathy and weight loss in nursing home residents: longitudinal study. J Am Med Dir Assoc. 2013;14(6):417-20.

51. Beaudart C, Sanchez-Rodriguez D, Locquet M, et al. Malnutrition as a Strong Predictor of the Onset of Sarcopenia. Nutrients. 2019;1 1(12):2883. 
52. Hai S, Cao L, Yang X, et al. Association Between Nutrition Status and Cognitive Impairment Among Chinese Nonagenarians and Centenarians. Int J Gerontol. 2017;11(4):215-9.

53. Kimura A, Sugimoto T, Kitamori K, et al. Malnutrition is Associated with Behavioral and Psychiatric Symptoms of Dementia in Older Women with Mild Cognitive Impairment and Early-Stage Alzheimer's Disease. Nutrients. 2019;11(8):1951.

54. Chow LS, Albright RC, Bigelow ML, et al. Mechanism of insulin's anabolic effect on muscle: measurements of muscle protein synthesis and breakdown using aminoacyl-tRNA and other surrogate measures. Am J Physiol Endocrinol Metab. 2006;291(4):E729-736.

55. Ansari SA, Emerald BS. The Role of Insulin Resistance and Protein O-GlcNAcylation in Neurodegeneration. Front Neurosci. 2019;13:473.

56. Burns JM, Donnelly JE, Anderson HS, et al. Peripheral insulin and brain structure in early Alzheimer disease. Neurology. 2007;69(11):1094-104.

57. Burns JM, Johnson DK, Watts A, et al. Reduced lean mass in early Alzheimer disease and its association with brain atrophy. Arch Neurol. 2010;67(4):428-33.

58. Alemán H, Esparza J, Ramirez FA, et al. Longitudinal evidence on the association between interleukin- 6 and C-reactive protein with the loss of total appendicular skeletal muscle in free-living older men and women. Age Ageing. 2011;40(4):469-75.

59. Sathyan S, Barzilai N, Atzmon G, et al. Association of anti-inflammatory cytokine IL10 polymorphisms with motoric cognitive risk syndrome in an Ashkenazi Jewish population. Neurobiol Aging. 2017;58:238.e231-238. e238.

60. Eurelings $L S$, Richard E, Eikelenboom P, et al. Low-grade inflammation differentiates between symptoms of apathy and depression in communitydwelling older individuals. Int Psychogeriatr. 2015;27(4):639-47.

61. Alosco ML, Spitznagel MB, Cohen R, et al. Decreased physical activity predicts cognitive dysfunction and reduced cerebral blood flow in heart failure. J Neurol Sci. 2014;339(1-2):169-75.

62. Kim S, Choi JY, Moon S, et al. Roles of myokines in exerciseinduced improvement of neuropsychiatric function. Pflugers Arch. 2019:471(3):491-505.

63. Scisciola L, Fontanella RA, et al. Sarcopenia and Cognitive Function: Role of Myokines in Muscle Brain Cross-Talk. Life (Basel). 2021;11(2):173.

\section{Publisher's Note}

Springer Nature remains neutral with regard to jurisdictional claims in published maps and institutional affiliations.

Ready to submit your research? Choose BMC and benefit from:

- fast, convenient online submission

- thorough peer review by experienced researchers in your field

- rapid publication on acceptance

- support for research data, including large and complex data types

- gold Open Access which fosters wider collaboration and increased citations

- maximum visibility for your research: over $100 \mathrm{M}$ website views per year

At BMC, research is always in progress.

Learn more biomedcentral.com/submissions 